\title{
Efficient Calculation of the Intramolecular Contribution to Orientational Autocorrelation Functions Using Dynamic Rotational Isomeric State Theory
}

\author{
Ivet Bahar \\ Department of Chemical Engineering and Polymer Research Center, Bogazici \\ University, Bebek 80815, Istanbul, Turkey \\ Wayne L. Mattice* \\ Institute of Polymer Science, The University of Akron, Akron, Ohio 44325-3909 \\ Received August 24, 1989; Revised Manuscript Received December 1, 1989
}

\begin{abstract}
A matrix formulation of the time-dependent transition partition function is combined with a generator matrix formalism to permit rapid and accurate calculation of the first and second orientation autocorrelation functions, $\langle\mathbf{m}(0) \cdot \mathbf{m}(\tau)\rangle$ and $\left\langle(3 / 2)(\mathbf{m}(0) \cdot \mathbf{m}(\tau))^{2}-1 / 2\right\rangle$, for a chain molecule. Here $\mathbf{m}$ is a unit vector rigidly attached to a bond in the chain, and 0 and $\tau$ denote this vector at times 0 and $\tau$. The time-dependent a priori probabilities of the type $p^{*}\left(x ; x_{0}\right)$, which denotes the probability that a bond $i$ in state $x$ at time 0 is still in state $x$ at time $\tau$, reduce correctly to those obtained from conventional equilibrium rotational isomeric state theory at zero and infinite times.
\end{abstract}

\section{Introduction}

Recently a matrix multiplication method has been proposed to describe the stochastics of conformational transitions between isomeric states in polymer chains. ${ }^{1}$ The chain is idealized as a microcanonical system where the relaxational motions are assumed to be determined by short-range intramolecular conformational potentials exclusively. This approach is somewhat equivalent to the classical treatment of the equilibrium statistics of an ideal chain, in the absence of excluded volume and/or specific solvent effects. ${ }^{2}$

In analogy with the conventional treatment of chain statistics where discrete rotational isomeric states are assigned statistical weights on the basis of the nearestneighbor interactions along the chain, ${ }^{2}$ stochastic weights can be defined ${ }^{1}$ to determine the probabilistic occurrence of transitions among those states. Suitable organization of the stochastic weights in a matrix formalism and serial multiplication following the usual methods of statistical mechanics yield the so-called transition partition function governing the stochastics of conformational transitions at a given time.

The approach developed by Bahar ${ }^{1}$ permits the evaluation of the joint probability of occurrence of any two configurations with a given time interval. Thus, the theory is readily applicable to the analysis of specific conformational transitions. In the present work the formulation is extended to include the evaluation of a dynamic quantity of interest from the experimental point of view, the orientational autocorrelation function (OACF) associated with a vectorial quantity in the chain.

In the following section, a brief recapitulation of the dynamic rotational isomeric states (DRIS) approach, ${ }^{3-5}$ which is the fundamental model underlying the present work, will be given, with emphasis on its application to the determination of OACFs. The new analytical method for the calculation of the OACF will be introduced in section III. It is followed by illustrative calculations and discussion in the last two sections.

\section{The DRIS Model and Its Application to Calculation of the OACF}

A. Time-Delayed Joint Probabilities. In the DRIS model, the type of conformational transitions accessible to interdependent pairs of bonds and the corresponding activation energies are estimated from short-range intramolecular energetic considerations. Conformational energy maps constructed as a function of two consecutive bond rotations are consulted for that purpose. Kramer's high friction limit rate expressions are used for the associated rate constants, with the front factor being left as an adjustable parameter inversely proportional to the effective viscosity. The rate constant associated with the passage from the $j^{\text {th }}$ state to the $i^{\text {th }}$ state constitutes the $i j^{\text {th }}$ element of the transition rate matrix $A^{(2)}$ governing the conformational kinetics of interdependent pairs of bonds. The diagonal elements of $A^{(2)}$ are determined from the microscopic reversibility requirement that the elements in each column should sum up to zero.

Let $p\left(\alpha \beta ; \alpha^{\circ} \beta^{\circ}\right)$ denote the joint probability of occurrence of two configurations $\alpha \beta$ and $\alpha^{\circ} \beta^{\circ}$, with a time interval $\tau$, for a pair of interdependent bonds. This quantity may conveniently be viewed as the $i j^{\text {th }}$ element of the symmetric time-delayed joint probability matrix $\mathcal{P}(\tau)$, provided that the set of states $\alpha \beta$ and $\alpha^{\circ} \beta^{\circ}$ are identified as the $i^{\text {th }}$ and $j^{\text {th }}$ configuration of the pair. Thus for a chain with three isomeric states trans $(t)$, gauche ${ }^{+}\left(\mathrm{g}^{+}\right)$, and gauche $e^{-}\left(\mathrm{g}^{-}\right)$available to each bond, $\mathcal{P}(\tau)$ is a $9 \times 9 \mathrm{ma}-$ trix consisting of the elements $p(\mathrm{tt} ; \mathrm{tt}), p\left(\mathrm{tt} ; \mathrm{tg}^{+}\right), \ldots$, $p\left(\mathrm{~g}^{-} \mathrm{g}^{-} ; \mathrm{g}^{-} \mathrm{g}^{-}\right)$in reading order. In the more general case where there are $\mu$ rotational isomers at each bond, the dimensions of $P(\tau)$ are $\mu^{2} \times \mu^{2}$. $P(\tau)$ is readily evaluated from the transition rate matrix $A^{(2)}$ by using the equation ${ }^{3,4}$

$$
\mathscr{P}(\tau)=\mathscr{B} \exp \{\Lambda \tau\} \mathcal{B}^{-1} \operatorname{diag}(\mathbf{P}(0))
$$

where $\Lambda$ is the diagonal matrix of the eigenvalues $\lambda_{1}, \lambda_{2}$, ..., $\lambda_{9}$ of $A^{(2)}, \mathscr{B}$ and its inverse $B^{-1}$ are the matrices of the eigenvectors and eigenrows following the transformation

$$
A^{(2)}=\mathscr{B} \Lambda \mathscr{B}^{-1}
$$

and diag $(\mathbf{P}(0))$ is the diagonal matrix of the elements $p^{\circ}(\mathrm{tt}), p^{\circ}\left(\mathrm{tg}^{+}\right), \ldots, p^{\circ}\left(\mathrm{g}^{-} \mathrm{g}^{-}\right)$of the probability vector $\mathbf{P}(0)$ of the initial occupancies of the various states. 
An equation similar to eq 1 may equally well be written for independent bonds, by using the $3 \times 3$ transition rate matrix $A^{(1)}$ valid for single bond independent dynamics. Clearly, $P(\tau)$ reduces in this case to a $3 \times 3$ matrix with the elements denoted as $p(\mathrm{t} ; \mathrm{t}), p\left(\mathrm{t} ; \mathrm{g}^{+}\right), \ldots, p\left(\mathrm{~g}^{-} ; \mathrm{g}^{-}\right)$.

B. Orientational Autocorrelation Functions. Let us consider a unit vectorial quantity $\mathbf{m}$ rigidly affixed to a chain. Following the Brownian motion of that chain, the orientation of $\mathbf{m}$ in space will change with time. A measure of the rate of reorientation is the OACF associated with $\mathbf{m}$. The first and second OACFs are defined as

$$
M_{1}(\tau)=\langle\mathbf{m}(0) \cdot \mathbf{m}(\tau)\rangle
$$

and

$$
M_{2}(\tau)=\left\langle(3 / 2)(\mathbf{m}(0) \cdot \mathbf{m}(\tau))^{2}-1 / 2\right\rangle
$$

respectively. Here the angular brackets refer to the ensemble average over all conformational transitions at time $\tau$. Clearly, $\mathbf{m}(0)$ and $\mathbf{m}(\tau)$ depend on the sets of isomeric states $\left\{\alpha^{\circ} \beta^{\circ} \gamma^{\circ} \ldots\right\}$ and $\{\alpha \beta \gamma \ldots\}$ characterizing, respectively, the initial and final configurations of the chain. If the functions in brackets in eqs 3 and 4 are shown as $f_{i}\left(\alpha \beta \gamma \ldots ; \alpha^{\circ} \beta^{\circ} \gamma^{\circ} \ldots\right)$, with $i=1$ and 2 , the OACF may be found from

$$
M_{i}(\tau)=\sum_{\alpha} \sum_{\beta} \sum_{\gamma} \ldots \sum_{\alpha^{\circ}} \sum_{\beta^{\circ}} \sum_{\gamma^{\circ}} \ldots f_{i}\left(\alpha \beta \gamma \ldots ; \alpha^{\circ} \beta^{\circ} \gamma^{\circ}\right) \times
$$

where $p\left(\alpha \beta \gamma \ldots ; \alpha^{\circ} \beta^{\circ} \gamma^{\circ} \ldots\right)$ is the joint probability of occurrence of the configurations $\left\{\alpha^{\circ} \beta^{\circ} \gamma^{\circ} \ldots\right\}$ and $\{\alpha \beta \gamma\}$ with a time interval $\tau$. The summations in eq 5 are performed over all the initial and final states of the rotatable bonds in the chain.

Two different approaches have been undertaken to compute $M_{i}(\tau)$ in previous work. In both approaches, complete enumeration technique has been employed. They differ in the procedure to evaluate the joint probability $p\left(\alpha \beta \gamma \ldots ; \alpha^{\circ} \beta^{\circ} \gamma^{\circ} \ldots\right)$. They may be outlined as follows:

(a) Bahar and Erman proposed ${ }^{4}$ an expression of the form

$$
\begin{aligned}
& p\left(\alpha \beta \gamma \delta \ldots ; \alpha^{\circ} \beta^{\circ} \gamma^{\circ} \delta^{\circ} \ldots\right)= \\
& \quad p\left(\alpha \beta ; \alpha^{\circ} \beta^{\circ}\right) q\left(\beta \gamma ; \beta^{\circ} \gamma^{\circ}\right) q\left(\gamma \delta ; \gamma^{\circ} \delta^{\circ}\right) \ldots
\end{aligned}
$$

where

$$
q\left(\beta \gamma ; \beta^{\circ} \gamma^{\circ}\right) \equiv p\left(\beta \gamma ; \beta^{\circ} \gamma^{\circ}\right) / \sum_{\gamma} \sum_{\gamma^{\circ}} p\left(\beta \gamma ; \beta^{\circ} \gamma^{\circ}\right) \ldots
$$

This expression is reminiscent of the doublet closure ${ }^{6}$ that is used for the analysis of the equilibrium state of the linear Ising model. Here, joint events of occupancies of pairs of states with a time interval $\tau$ are treated.

(b) By suitable combination of the transition rate matrices $A^{(2)}$ for pairs of bonds, in direct product formalism, an expression was derived by Bahar, Erman, and Monnerie ${ }^{5}$ for the transition rate matrix $A^{(N)}$ governing the conformational kinetics of $N$ pairwise interdependent mobile bonds. Similarity transformation of the latter and substitution of the resulting matrices in eq 1 yield the joint probability matrix of size $3^{N} \times 3^{N}$ for $N$ mobile bonds. This procedure requires the diagonalization of a matrix of order $3^{N}$ and consequently is limited to smaller $N(N \leq 5)$ compared to approach a, whereas the method in approach a may be extended to longer sequences of bonds at the expense of computation time only. On the other hand, approach $b$ has the advantage of yield-

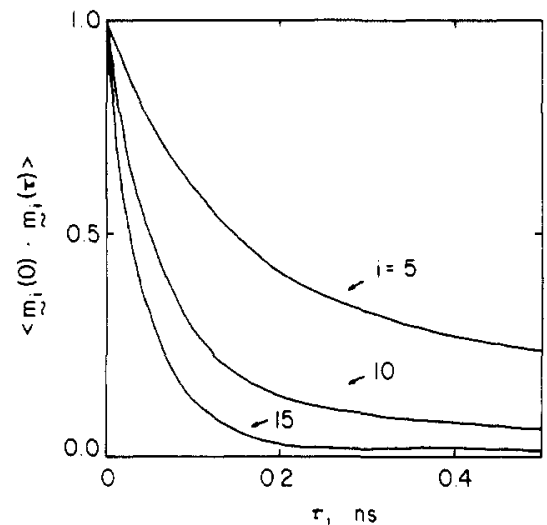

Figure 1. First orientational autocorrelation function at 300 $\mathrm{K}$ for bond $i$ in a polyethylene chain of 20 bonds.

ing the $3^{N}$ eigenvalues that are representative of the frequencies of the $3^{N}-1$ modes contributing to relaxation (one of them being equal to zero). Thus, in this case, the decay of the OACF with time is conveniently written as

$$
M_{i}(\tau)=\sum_{j=1}^{3^{N}} k_{j} \exp \left\{\lambda_{j} \tau\right\}
$$

where the time-independent amplitude factors $k_{i}$ are found for the specific type of correlation function $f_{i}$ by using $\mathscr{B}, \mathcal{B}^{-1}, \Lambda$, and $\mathbf{P}(0) .^{5}$

Calculations performed for mobile segments in polyethylene (PE) using either method a or b yield almost indistinguishable decay curves for the OACFs, confirming the equivalence of the two approaches. However, both of them differ from the limitations of the complete enumeration technique, and satisfactory comparison of the DRIS formalism with Brownian dynamics simulations and experimental and theoretical works on the highfrequency motions ${ }^{5,7,8}$ cells for a more rigorous analytical method for calculating the OACFs. The latter is presented in the next section.

\section{Matrix Multiplication Scheme To Compute OACFs}

According to the recently developed treatment of conformational stochastics in a chain of $N$ bonds, a transition partition function $Z(\tau)$ is defined for a given time by $^{1}$

$$
Z(\tau)=\mathbf{J}^{\mathrm{T}}\left[\prod_{i=2}^{N-1} \mathbf{V}_{i}(\tau)\right] \mathbf{J}
$$

where $V_{i}(\tau)$ is the stochastic weight matrix corresponding to the pair of bonds $i-1$ and $i, \mathbf{J} \equiv \operatorname{col}(1,1, \ldots, 1)$, and $\mathbf{J}^{\mathrm{T}} \equiv$ row $(1,1, \ldots, 1)$. For a chain with three isomeric states $t, g^{+}$, and $g^{-}$accessible to each bond, the stochastic weight matrices read

$\mathbf{V}_{2}(\tau)=\left[\begin{array}{ccccc}p(\mathrm{t} ; \mathrm{t}) & & & & \\ & p\left(\mathrm{t} ; \mathrm{g}^{+}\right) & & & \\ & & p\left(\mathrm{t} ; \mathrm{g}^{-}\right) & & \\ & & & \ldots & \\ & & & & p\left(\mathrm{~g}^{-} ; \mathrm{g}^{-}\right)\end{array}\right]$

and $V_{i}$ for $2<i \leq N-1$ is composed of 9 block elements, each corresponding to a fixed state at time $\tau$, according to 


$$
\mathbf{V}_{i}(\tau)=\left[\begin{array}{lllllll}
v(\mathrm{tt} ; \mathrm{tt}) & v\left(\mathrm{tt} ; \mathrm{tg}^{+}\right) & v\left(\mathrm{tt} ; \mathrm{tg}^{-}\right) & v\left(\mathrm{tg}^{+} ; \mathrm{tt}\right) & v\left(\mathrm{tg}^{+} ; \mathrm{tg}^{+}\right) & v\left(\mathrm{tg}^{+} ; \mathrm{tg}^{-}\right) & \ldots \\
v\left(\mathrm{tt} ; \mathrm{g}^{+} \mathrm{t}\right) & v\left(\mathrm{tt} ; \mathrm{g}^{+} \mathrm{g}^{+}\right) & v\left(\mathrm{tt} ; \mathrm{g}^{+} \mathrm{g}^{-}\right) & v\left(\mathrm{tg}^{+} ; \mathrm{g}^{+} \mathrm{t}\right) & \ldots & & \\
v\left(\mathrm{tt} ; \mathrm{g}^{-} \mathrm{t}\right) & v\left(\mathrm{tt} ; \mathrm{g}^{-} \mathrm{g}^{+}\right) & v\left(\mathrm{tt} ; \mathrm{g}^{-} \mathrm{g}^{-}\right) & \ldots & & & \\
v\left(\mathrm{~g}^{+} \mathrm{t} ; \mathrm{tt}\right) & v\left(\mathrm{~g}^{+} \mathrm{t} ; \mathrm{tg}^{+}\right) & v\left(\mathrm{~g}^{+} \mathrm{t} ; \mathrm{tg}^{-}\right) & \ldots & & \\
v\left(\mathrm{~g}^{+} \mathrm{t} ; \mathrm{g}^{+} \mathrm{t}\right) & v\left(\mathrm{~g}^{+} \mathrm{t} ; \mathrm{g}^{+} \mathrm{g}^{+}\right) & v\left(\mathrm{~g}^{+} \mathrm{t} ; \mathrm{g}^{+} \mathrm{g}^{-}\right) & \ldots & & \\
v\left(\mathrm{~g}^{+} \mathrm{t} ; \mathrm{g}^{-} \mathrm{t}\right) & v\left(\mathrm{~g}^{+} \mathrm{t} ; \mathrm{g}^{-} \mathrm{g}^{+}\right) & v\left(\mathrm{~g}^{+} \mathrm{t} ; \mathrm{g}^{-} \mathrm{g}^{-}\right) & \ldots & & \\
v\left(\mathrm{~g}^{-} \mathrm{t} ; \mathrm{tt}\right) & \ldots & & & & \\
v\left(\mathrm{~g}^{-} ; \mathrm{g}^{+} \mathrm{t}\right) & \ldots & & & & \\
v\left(\mathrm{~g}^{-} \mathrm{t} ; \mathrm{g}^{-} \mathrm{t}\right) & \ldots & & & & v\left(\mathrm{~g}^{-} \mathrm{g}^{-} ; \mathrm{g}^{-} \mathrm{g}^{-}\right)
\end{array}\right]_{i}
$$

where

$$
v(\alpha \beta ; \gamma \delta) \text { घ } p(\alpha \beta ; \gamma \delta) / p(\alpha ; \gamma)
$$

It should be recalled that $p(\alpha \beta ; \gamma \delta)$ and $p(\alpha ; \gamma)$ refer to the time-delayed joint probabilities for pairwise-dependent and -independent bonds, respectively.

The above matrices may conveniently be employed to determine the a priori probabilities of specific isomeric transitions at a given time. ${ }^{1}$ In fact

$$
p_{j}^{*}\left(\mathrm{t} ; \mathrm{g}^{+}\right)=[Z(\tau)]^{-1} \mathbf{J}^{\mathrm{T}}\left[\prod_{i=2}^{j-1} \mathbf{V}_{i}(\tau)\right] \mathbf{V}_{j}^{*}(\tau)\left[\prod_{i=j+1}^{N-1} \mathbf{V}_{i}(\tau)\right] \mathbf{J}
$$

yields the a priori probability of occurrence of states $t$ and $\mathrm{g}^{+}$with a time interval $\tau$ for the $j^{\text {th }}$ bond along the chain. Here, $V_{j}^{*}(\tau)$ is the stochastic weight matrix where all elements $v_{j}(\alpha \beta ; \gamma \delta)$ with $\beta \neq \mathrm{t}$ and $\delta \neq \mathrm{g}^{+}$are equated to zero. Similar expressions may be written for different conformational states and/or larger numbers of consecutive bonds.

Let us now consider the first $\mathrm{OACF}$ associated with a vector $\mathbf{m}$ affixed to a polymer at a fixed location. A reference frame of observation may be chosen as the one whose $x$ axis coincides with the first bond of the chain, the $y$ axis makes an acute angle with the prolongation of a hypothetical preceding bond in the trans state, and the $z$ axis completes a right-handed coordinate system. This defines the convenient bond-based frame $\mathrm{e}^{2}$ associated with bond 1 . The investigated vector $\mathbf{m}$ will be assumed to be rigidly embedded in the bond-based frame of bond $i$ +1 . Let $m^{\circ}$ be its representation in that local frame. Then the first OACF may be written as

$$
\begin{aligned}
M_{1}(\tau) & =\left\langle\left(\mathbf{T}(0) \mathbf{m}^{\circ}\right) \cdot\left(\mathbf{T}(\tau) \mathbf{m}^{\circ}\right)\right\rangle \\
= & \mathbf{m}^{\circ \mathrm{T}}\left\langle\mathbf{T}^{\mathrm{T}}(0) \mathbf{T}(\tau)\right\rangle \mathbf{m}^{\circ}
\end{aligned}
$$

where $\mathbf{T}$ is the transformation matrix that operates between bond-based frames $i+1$ and 1 . It is given by the serial product $\mathbf{T}_{1} \mathbf{T}_{2} \mathbf{T}_{3} \ldots \mathbf{T}_{i}$ of the conventional transformation matrices ${ }^{2}$ between two consecutive frames. The time argument follows from the dependence of the individual $\mathbf{T}_{j}$ 's, $1<j \leq i$, on the instantaneous torsional angles. The definition of the reference frame for observation requires that $\mathbf{T}_{1}$ be time independent; i.e., $\phi_{1}$ is defined to have the value for a $t$ state. The superscript $T$ denotes the transpose of the matrix. The ensemble average in brackets in eq 15 may be rewritten as

$$
\left\langle\mathbf{T}^{\mathrm{T}}(0) \mathbf{T}(\tau)\right\rangle=\left\langle\left(\prod_{q=1}^{i} \mathbf{T}_{q}(0)\right)^{\mathbf{T}}\left(\prod_{q=1}^{i} \mathbf{T}_{q}(\tau)\right)\right\rangle
$$

Thus, the problem of finding the OACF reduces to the evaluation of the average quantity on the right-hand side of eq 16. For this purpose, we recall that for any three conformable matrices $\mathbf{A}, \mathbf{B}$, and $\mathbf{C}$, a relationship of the form

$$
\{\mathrm{ABC}\}_{\mathrm{col}}=\left(\mathbf{A} \otimes \mathbf{C}^{\mathrm{T}}\right)\{\mathbf{B}\}_{\mathrm{col}}
$$

may be written. Here, \{\}$_{\text {col }}$ denotes the column array of the elements of the matrix enclosed therein, the elements being arranged in usual reading order, and $\otimes$ signifies the direct matrix product. This identity was employed by Jernigan and Flory ${ }^{9}$ in the RIS treatment of the optical anisotropy of chain molecules. Likewise, it may be demonstrated that

$$
\{A B C\}_{\text {row }}=\{B\}_{\text {row }}\left(A^{\mathrm{T}} \otimes \mathbf{C}\right)
$$

where \{\}$_{\text {row }}$ is the transpose of \{\}$_{\text {col }}$. Identification of $\mathbf{A}$ with $\left[\prod_{q=1}^{i} \mathbf{T}_{q}(0)\right]$ and $\mathbf{C}$ with $\left[\prod_{q=1}^{i} \mathbf{T}_{q}(\tau)\right]$ and substitution of the identity matrix $\mathbf{E}_{3}$ of order 3 for $\mathbf{B}$ yields

$$
\begin{aligned}
&\left\{\left\langle\left[\prod_{q=1}^{i} \mathbf{T}_{q}(0)\right]^{\mathrm{T}}\left[\prod_{q=1}^{i} \mathbf{T}_{q}(\tau)\right]\right\rangle\right\}_{\text {row }}= \\
&\left\{\mathbf{E}_{3}\right\}_{\text {row }}\left\langle\left[\prod_{q=1}^{i} \mathbf{T}_{q}(0)\right] \otimes\left[\prod_{q=1}^{i} \mathbf{T}_{q}(\tau)\right]\right\rangle \\
&=\left\{\mathbf{E}_{3}\right\}_{\text {row }}\left\langle\prod_{q=1}^{i}\left[\mathbf{T}_{q}(0) \otimes \mathbf{T}_{q}(\tau)\right]\right\rangle
\end{aligned}
$$

Equation 20 follows from eq 19 upon an appeal to the theorem on direct products. ${ }^{10}$ The product in eq 20 is similar to the one encountered ${ }^{11,12}$ in the treatment of the fourth moment of the end-to-end vector $\mathbf{r}$ in polymer chains. The major difference is that here the two transformation matrices correspond to different times.

In order to write the expression in the form of eq 15 , it is necessary to reexpress the row of nine elements in eq 20 as a $3 \times 3$ matrix. This objective is obtained by performing the following operation.

$$
\begin{gathered}
M_{1}(\tau)=\mathbf{m}^{\circ \mathrm{T}}\left(\mathbf{E}_{3} \otimes \mathbf{F}\right)\left(\mathbf{D}^{\mathrm{T}} \otimes \mathbf{E}_{3}\right) \mathbf{m}^{\circ} \\
\mathbf{D}=\left\{\mathbf{E}_{3}\right\}_{\text {row }}\left(\prod_{q=1}^{i}\left[\mathbf{T}_{q}(0) \otimes \mathbf{T}_{q}(\tau)\right]\right\rangle \\
\mathbf{F}=\operatorname{row}(1,0,0,0,1,0,0,0,1)
\end{gathered}
$$

In analogy to the matrix multiplication scheme of equilibrium statistics, the average in eq 20 may be found from

$$
\begin{aligned}
& \left\langle\prod_{q=1}^{i}\left[\mathbf{T}_{q}(0) \otimes \mathbf{T}_{q}(\tau)\right]\right\rangle= \\
& \quad[Z(\tau)]^{-1}\left[\left(\mathbf{J}^{\mathbf{T}} \otimes \mathbf{E}_{9}\right)\left[\left(\mathbf{V} \otimes \mathbf{E}_{g}\right)\left\|\mathbf{T}_{q}(0) \otimes \mathbf{T}_{q}(\tau)\right\|\right]_{2}^{(i-1)} \times\right. \\
& \left.\quad\left(\mathbf{V} \otimes \mathbf{E}_{9}\right)_{i+1}^{(N-i-1)}\left(\mathbf{J} \otimes \mathbf{E}_{9}\right)\right]
\end{aligned}
$$

where $\mathbf{E}_{9}$ is the identity matrix of order 9 . The notation ()$_{i}^{(j)}$ signifies the serial product of $j$ factors of the quantity so designated, the initial factor possessing serial index 
i. II $\|$ is an operator denoting the diagonal supermatrix such that

$$
\left\|\mathbf{T}_{q}(0) \otimes \mathbf{T}_{q}(\tau)\right\|=
$$

$$
\mathrm{T}_{\mathrm{t}} \otimes \mathrm{T}_{\mathrm{g}^{+}} \quad \mathrm{T}_{\mathrm{t}} \otimes \mathrm{T}_{\mathrm{g}^{-}}
$$

$\mathbf{T}_{\mathrm{t}}, \mathbf{T}_{\mathbf{g}^{+}}$, and $\mathbf{T}_{\mathbf{g}}$ are the transformation matrices where the torsional angles for the trans, gauche ${ }^{+}$, and gauche ${ }^{-}$ states are inserted, respectively. The product of the supermatrices of order 81 for bonds 1 through $N-i-1$ in eq 25 is reduced to a matrix of order 9 in eq 24 by premultiplication by $\left(\mathbf{J}^{\mathrm{T}} \otimes \mathbf{E}_{9}\right)$ and postmultiplication by $(\mathbf{J} \otimes$ $\left.\mathbf{E}_{9}\right)$. The resulting average $9 \times 9$ matrix is transformed into a row of 9 elements in eq 20 , and inserted into eq 15 , as the identity in eq 16 implies. In this final matrix $\left\langle\mathbf{T}^{\mathrm{T}}(0) \mathbf{T}(\tau)\right\rangle$, the diagonal elements refer to the first OACF for the vectors along the $x, y$, and $z$ axes of the bondbased frame $i+1$, while the off-diagonal terms represent the orientational cross correlations between the three axial vectors.

An approach similar to the one described above for the first OACF may be used to derive an analytical expression for the second OACF as well. The relevant average quantity in the latter is $\left\langle(\mathbf{m}(0) \cdot \mathbf{m}(\tau))^{2}\right\rangle$, which may be written as 2,12

$$
\begin{aligned}
& \left\langle(\mathbf{m}(0) \cdot \mathbf{m}(\tau))^{2}\right\rangle= \\
& \left\langle\left[\mathbf{m}^{\circ \mathrm{T}} \mathbf{T}^{\mathrm{T}},(0) \mathbf{T}(\tau) \mathbf{m}^{\circ}\right] \otimes\left[\mathbf{m}^{\circ \mathrm{T}} \mathbf{T}^{\mathrm{T}}(0) \mathbf{T}(\tau) \mathbf{m}^{\circ}\right]\right\rangle \\
& =\left\langle\left(\mathbf{m}^{\circ \mathrm{T}} \otimes \mathbf{m}^{\circ \mathrm{T}}\right)\left(\mathbf{T}^{\mathrm{T}}(0) \otimes \mathbf{T}^{\mathrm{T}}(0)\right)(\mathbf{T}(\tau) \otimes \mathbf{T}(\tau)) \times\right. \\
& \left.\left(\mathbf{m}^{\circ} \otimes \mathbf{m}^{\circ}\right)\right\rangle
\end{aligned}
$$

Recognizing the $\mathrm{m}^{\circ \mathrm{T}}$ and $\mathrm{m}^{\circ}$ are constant, using the identity $\mathbf{A}^{\mathrm{T}} \otimes \mathbf{A}^{\mathrm{T}}=(\mathbf{A} \otimes \mathbf{A})^{\mathrm{T}}$, and substituting $\mathbf{S}(\tau)$ for $(\mathrm{T}(\tau)$ $\otimes T(\tau))$, we may write eq 27 as

$$
\left\langle(\mathbf{m}(0) \cdot \mathbf{m}(\tau))^{2}\right\rangle=\left(\mathbf{m}^{\circ \mathrm{T}} \otimes \mathbf{m}^{\circ \mathrm{T}}\right)\left\langle\mathbf{S}^{\mathrm{T}}(0) \mathbf{S}(\tau)\right\rangle\left(\mathbf{m}^{\circ} \otimes \mathbf{m}^{\circ}\right)
$$

It is noted that eq 28 is similar in form to eq 15 . The calculation of the average quantity in eq 28 may be performed, therefore, by following exactly the same procedure as outlined in detail above. It suffices to replace each $\mathbf{T}_{q}$ either at $t=0$ or $\tau$ by $\mathbf{T}_{q} \otimes \mathbf{T}_{q}$ in eqs 20 and 24 . The elements of the diagonal supermatrix are accordingly rearranged such that, for instance, the second block element on the diagonal becomes $\left(\mathbf{T}_{\mathrm{t}} \otimes \mathbf{T}_{\mathrm{t}}\right) \otimes\left(\mathbf{T}_{\mathbf{g}^{+}} \otimes \mathbf{T}_{\mathrm{g}^{+}}\right)$.

\section{Calculations}

To test the validity of the analytical solution given by eqs $18-25$, coupled with eqs 15 and 16 , the decay of the first OACF with time was calculated for various bonds $i$ along a 20-bond PE chain. The same data as in the previous calculations are used ${ }^{4}$ with the exception of $E_{\sigma}=$ $0.6 \mathrm{kcal} / \mathrm{mol}$ for the energy of the $\mathrm{g}^{ \pm}$state in excess of the $t$ state. Inasmuch as the frame of observation moves with bond 1 as mentioned above, the OACF reflects the internal motions of the chain only. Curves for $i=5,10$, and 15 at $T=300 \mathrm{~K}$ are shown in Figure 1 . The fact that a faster decay occurs with larger $i$ arises from the higher degrees of freedom of the latter. Those results are valid for a medium where the same effective frictional resistance applies on each bond.

Similarly, the decrease in orientational correlations as the number of bonds between the frame of observation and the investigated bond increases is illustrated in Fig-

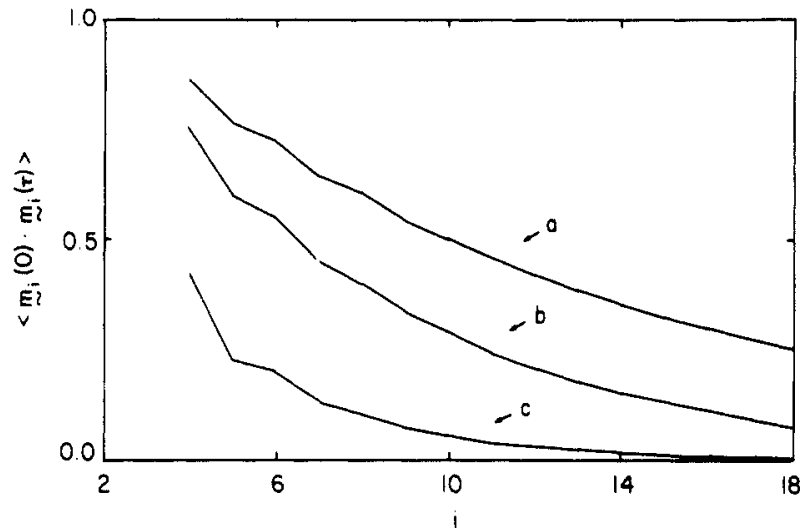

Figure 2. First orientational autocorrelation function at 300 $\mathrm{K}$ for bond $i$ in a polyethylene chain of 20 bonds at times of (a) 0.05 , (b) 0.1 , and (c) $0.5 \mathrm{~ns}$.

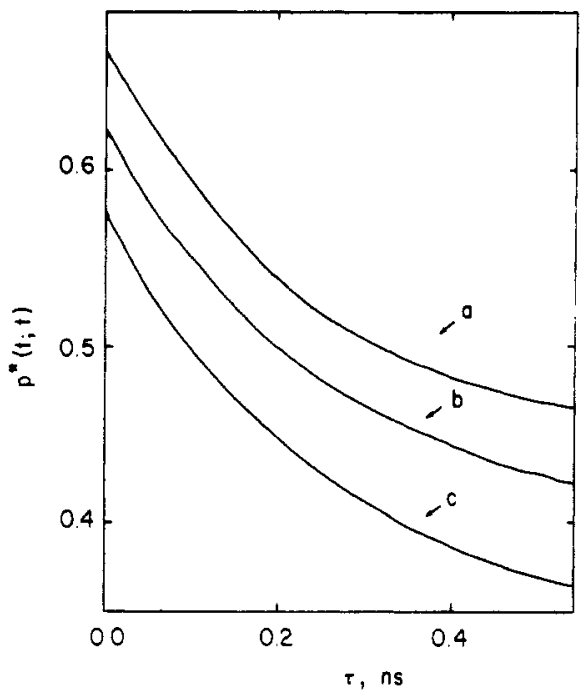

Figure 3. Joint probabilities for trans states at internal bonds in a polyethylene chain of 20 bonds at $300 \mathrm{~K}$ according to (a) the exact dynamic rotational isomeric state treatment of the present work, (b) the approach described as method a in section II, and (c) treating the bonds as being independent.

ure 2. Curves $\mathrm{a}, \mathrm{b}$, and $\mathrm{c}$, calculated for three distinct time intervals, show that the even-odd effect that is characteristic of the dynamics of short segments ${ }^{8,13}$ disappears as the size of the mobile segment increases.

It is noted that the curves in Figures 1 and 2 and similar calculations on OACFs reproduce very closely the results previously obtained for shorter segments, ${ }^{7,13}$ using either method a or b outlined in section II. The important aspect of the present calculations, however, is that now the calculation procedure, i.e., the analytical method, is incomparably faster than the previous complete enumeration technique. Besides, the extension to longer chains is easily carried out.

The OACFs, in a way, result from the superposition of several motions and are not very sensitive to the individual bond probabilities. A more thorough examination of the conformational stochastics of the bonds shows that if, in turn, the transitions of specific isomeric states are considered, the decay curves resulting from method a in section II differ from those predicted by the present matrix multiplication scheme. Figure 3 illustrates the decrease of $p^{*}(\mathrm{t} ; \mathrm{t})$ with time for an internal bond in a 20 -bond PE chain at $300 \mathrm{~K}$. The upper curve is the a priori joint probability obtained from an expression of the form of eq 13 . The middle curve results from method a in section II. The lowest curve corresponds to independent bonds. It is observed that the upper curve is the one 


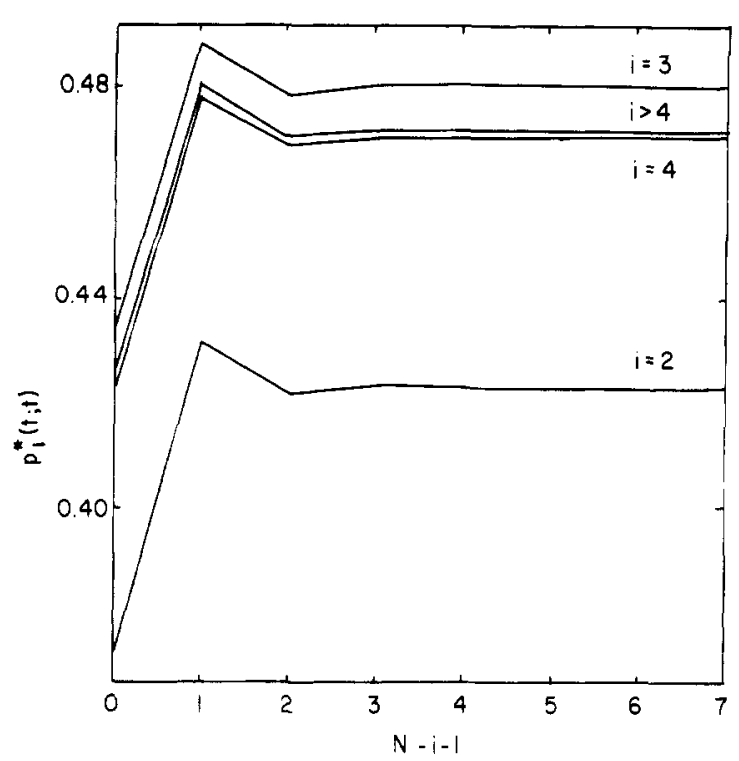

Figure 4. A priori joint probabilities $p_{i}^{*}(t ; t)$ in polyethylene chains at 0.5 ns and $300 \mathrm{~K}$ as a function of bond location $i$ and chain length $N$.

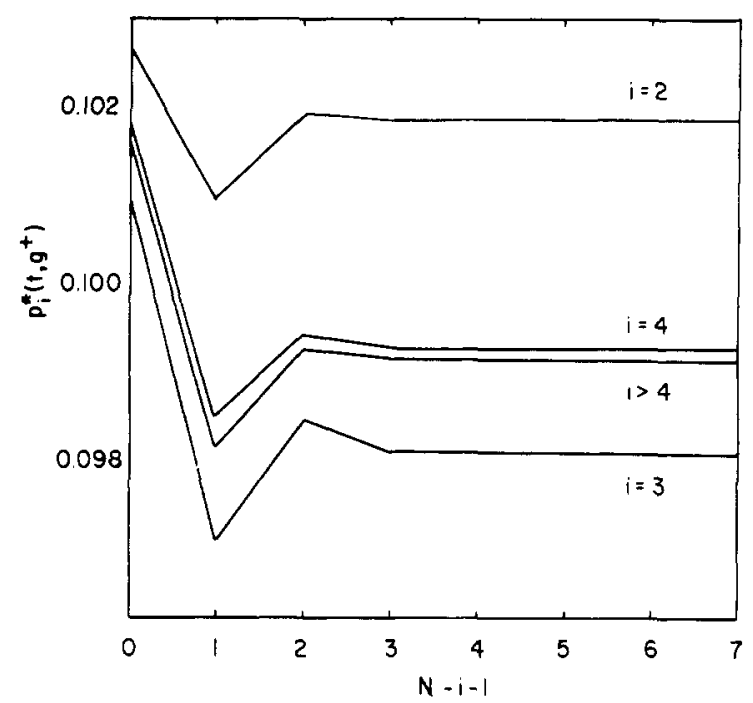

Figure 5. A priori joint probabilities $p_{i}^{*}\left(\mathrm{t} ; \mathrm{g}^{+}\right)$in polyethylene chains at $0.5 \mathrm{~ns}$ and $300 \mathrm{~K}$ as a function of bond location $i$ and chan length $N$.

that agrees in the two limits of $\tau=0$ and $\infty$, with the conventional equilibrium probabilities of the RIS model. Furthermore, at $\tau=0$, the lower curve is equal to $1 /(1$ $+2 \sigma$ ), which is smaller than the content of trans placements when $\mathrm{g}^{+} \mathrm{g}^{-}$and $\mathrm{g}^{-} \mathrm{g}^{+}$bond pairs are suppressed, as they are in the top curve.

The matrix multiplication scheme may be further used to evaluate, as in Figures 4 and 5 , the instantaneous a priori joint probabilities as a function of chain length and bond location. The horizontal axis is plotted as $N-$ $i-1$ in order to facilitate comparison with the equilibrium probabilities calculated by Jernigan and Flory. ${ }^{2,14}$ The curves correspond to $\tau=0.5 \mathrm{~ns}$. The joint probabilities exhibit an even-odd dependence on bond location, reminiscent of the predictions of the RIS model of chain statistics. In fact, Figure 4, for instance, represents the probability that a bond initially ir the trans state will have its state unchanged at a later time $\tau$. Thus, this probability is a measure of the residence time in the $t$ state. It is interesting to note that this property exactly parallels the equilibrium probability of occurrence of the trans state, ${ }^{2,14}$ as may be checked from the comparison of Figure 4 with the $p(\mathrm{t})$ vs $N-i-1$ curves obtained by the RIS formalism. This close similarity is not by coincidence, however. It reflects the well-known time ensemble equivalence of average conformational properties. It simply means that if a state has low equilibrium probability of occurrence, the period of time a given bond stays in that state is short, or vice versa. It should be noted that the approach of section II would invariably yield constant probabilities, i.e., horizontal lines, regardless of the bond serial order.

\section{v. Conclusion and Discussion}

The mathematical formalism of the present work presents a concise and systematic method of computing dynamic properties averaged over all conformational transitions of the chain. Similarly, pertinent analyses of events associated with specific conformational changes, such as helix-coil transition or cyclization phenomenon, may be performed with the aid of the present formulation. Clearly, direct quantitative correlation with experiments would require the proper incorporation of intermolecular, and intrachain but long-range, effects into the treatment. This may be achieved through suitable rescaling of the elements of the stochastic weight matrices, though the matrix multiplication scheme developed in section III preserves its validity.

An interesting aspect of the present formulation is to demonstrate, on a quantitative basis, the one-to-one correspondence between the static and dynamic probabilities of occupancies of various states. The even-odd dependence of the a priori transition probabilities on bond serial order along the chain is in exact conformity with the wellknown end effects of equilibrium statistics.

It is always possible to improve the theory through consideration of higher order closure approximation compared to the doublet closure presently adopted. The above formulation may be easily adapted to the triplet closure ${ }^{6}$ approximation. In this case, the doublet and singlet joint probabilities in eq 12 are replaced by triplet and doublet joint probabilities, respectively. This better approximation may be worth undertaking if accurate knowledge of solvent-mediated conformational energetics of groups of three consecutive bonds is available. Such an approach may be particularly useful inasmuch as correlations between second neighbors (i.e., bonds $i$ and $i+2$, for example) are asserted ${ }^{15}$ to be important in local conformational dynamics.

Acknowledgment. This research was supported by a grant from the Exxon Education Foundation.

\section{References and Notes}

(1) Bahar, I. J. Chem. Phys. 1989, 91, 6525.

(2) Flory, P. J. Statistical Mechanics of Chain Molecules; Interscience: New York, 1969. Reprinted by Hanser Publishers, Oxford University Press, 1989.

(3) Jernigan, R. L. In Dielectric Properties of Polymers; Karasz, F. E., Ed.; Plenum: New York, 1972; p 99.

(4) Bahar, I.; Erman, B. Macromolecules 1987, 20, 1368.

(5) Bahar, I.; Erman, B.; Monnerie, L. Macromolecules 1989, 22, 2396.

(6) Schwarz, G. Ber. Bunsenges. Phys. Chem. 1971, 75, 40.

(7) Bahar, I.; Erman, B.; Monnerie, L. Macromolecules 1989, 22, 431.

(8) Bahar, I.; Mattice, W. L. J. Chem. Phys. 1989, 90, 6775, 6783.

(9) Jernigan, R. L.; Flory, P. J. J. Chem. Phys. 1967, 47, 1999.

(10) According to the theorem on direct products, $(A \otimes B)(C \otimes D)$ $=(\mathbf{A C}) \otimes(\mathbf{B D})$, where $\mathbf{A}, \mathbf{B}, \mathbf{C}$, and $\mathbf{D}$ are appropriately conformable matrices.

(11) Nagai, K. J. Chem. Phys. 1963, 38, 924.

(12) Flory, P. J.: Jernigan, R. L. J. Chem. Phys, 1965, 42, 3509.

(13) Bahar, I.; Erman, B. J. Chem. Phys. 1988, 88, 1228.

(14) Jernigan, R. L.; Flory, P. J. J. Chem. Phys. 1969, 50, 4165.

(15) Helfand, E.; Wasserman, Z. R.; Weber, T. A. Macromolecules $1980,13,526$. 